\section{(C) OPEN ACCESS}

\title{
Sham surgery versus labral repair or biceps tenodesis for type II SLAP lesions of the shoulder: a three-armed randomised clinical trial
}

\author{
Cecilie Piene Schrøder, ${ }^{1}$ Øystein Skare, ${ }_{1}^{1}$ Olav Reikerås, ${ }^{2,3}$ Petter Mowinckel, ${ }^{2}$ \\ Jens Ivar Brox ${ }^{2,3}$
}

\begin{abstract}
- Additional material is published online only. To view please visit the journal online (http://dx.doi.org/10.1136/ bjsports-2016-097098)
\end{abstract}

1Orthopedic Department, Lovisenberg Diaconal Hospital, Oslo, Norway

${ }^{2}$ Oslo University Hospital, Oslo, Norway

${ }^{3}$ University of Oslo, Oslo, Norway

\section{Correspondence to} Dr Cecilie Piene Schrøder, Orthopedic Department, Lovisenberg Diaconal Hospital Lovisenberggt 17, 0440 Oslo, Norway; cecilie.schroder@lds.no

Accepted 10 March 2017 Published Online First 12 May 2017

\section{Linked}

http://dx.doi.org/10.1136/ bjsports-2017-097696

- http://dx.doi.org/10.1136/ bjsports-2017-098081

- http://dx.doi.org/10.1136/ bjsports-2017-098158

\section{CrossMark}

\section{To cite: Schrøder $C P$,}

Skare $\varnothing$, Reikerås 0 ,

et al. Br J Sports Med

2017:51:1759-1766.

\section{ABSTRACT}

Background Labral repair and biceps tenodesis are routine operations for superior labrum anterior posterior (SLAP) lesion of the shoulder, but evidence of their efficacy is lacking. We evaluated the effect of labral repair, biceps tenodesis and sham surgery on SLAP lesions.

Methods A double-blind, sham-controlled trial was conducted with 118 surgical candidates (mean age 40 years), with patient history, clinical symptoms and MRI arthrography indicating an isolated type II SLAP lesion. Patients were randomly assigned to either labral repair ( $n=40)$, biceps tenodesis $(n=39)$ or sham surgery $(n=39)$ if arthroscopy revealed an isolated SLAP II lesion. Primary outcomes at 6 and 24 months were clinical Rowe score ranging from 0 to 100 (best possible) and Western Ontario Shoulder Instability Index (WOSI) ranging from 0 (best possible) to 2100. Secondary outcomes were Oxford Instability Shoulder Score, change in main symptoms, EuroQol (EQ-5D and EQ-VAS), patient satisfaction and complications.

Results There were no significant between-group differences at any follow-up in any outcome. Betweengroup differences in Rowe scores at 2 years were: biceps tenodesis versus labral repair: $1.0(95 \% \mathrm{Cl}-5.4$ to 7.4), $p=0.76$; biceps tenodesis versus sham surgery: 1.6 $(95 \% \mathrm{Cl}-5.0$ to 8.1$), \mathrm{p}=0.64$; and labral repair versus sham surgery: $0.6(95 \% \mathrm{Cl}-5.9$ to 7.0$), p=0.86$. Similar results - no differences between groups - were found for WOSI scores. Postoperative stiffness occurred in five patients after labral repair and in four patients after tenodesis.

Conclusion Neither labral repair nor biceps tenodesis had any significant clinical benefit over sham surgery for patients with SLAP II lesions in the population studied.

Trial registration number ClinicalTrials.gov identifier: NCT00586742

\section{INTRODUCTION}

The superior glenoid labrum of the shoulder is a common site of injury and degeneration, which can cause both pain and disability. ${ }^{12}$ Snyder et al ${ }^{1}$ used the term SLAP (superior labrum anterior posterior) to describe a combined detachment of the long head of biceps tendon and the superior labrum from the glenoid rim (figure 1). The diagnosis is controversial; the validity of clinical tests, ${ }^{3}$ and even arthroscopy and MRI arthrography is questioned. ${ }^{4-8}$ In addition, a high prevalence of SLAP lesions has been reported in a middle-aged asymptomatic population. ${ }^{9}$
Labral repair is the most common procedure to treat labral tears, but because of high rates of complications and poor outcomes, ${ }^{10-17}$ it has been suggested that indications should be narrowed. ${ }^{10-17}$ Recent reports show a decrease in the number of labral tears performed and a decrease in the age of the patients undergoing these operations. ${ }^{18} 19$ Release of the biceps tendon (tenodesis or tenotomy) is increasingly used as an alternative ${ }^{1318-22}$ to SLAP repairs in select patients, but the evidence for it is weak. ${ }^{13}$ 20-22 One non-controlled study reported better results after tenodesis compared with labral repair, ${ }^{13}$ but no randomised trials have compared these procedures. Very little evidence about non-operative treatment for type II SLAP lesions was available when the present study was designed. Recent non-controlled studies suggest that non-operative treatment including graded exercise therapy is beneficial, and the postoperative rehabilitation in the present study followed those rehabilitation principles. $^{23-25}$

Our aim was to conduct a randomised, doubleblind, sham-controlled trial to assess the clinical effectiveness of labral repair and biceps tenodesis in patients with a type II SLAP lesion.

\section{MATERIALS AND METHODS \\ Design}

This blinded, three-armed randomised, sham-controlled study with a 2-year follow-up was conducted at Lovisenberg Diaconal Hospital, Oslo, Norway, from January 2008 to January 2014. The study protocol has been published previously. ${ }^{26}$ The patients, the treating physiotherapists/manual therapists and the persons collecting and analysing the data were blinded to the study group assignments. The first author was not blinded to group assignment, but had no role in the follow-up of the patients.

\section{Participants}

We enrolled patients $18-60$ years of age who had shoulder pain ( $>3$ months) that was unresponsive to conventional non-operative treatment (physiotherapy, non-steroidal anti-inflammatory drugs and/or corticosteroid injections) and had a history, clinical findings and MRI arthrography indicating a SLAP lesion. Patients were informed that final study inclusion and randomisation would be performed during arthroscopy if an isolated type II SLAP lesion was confirmed. Exclusion criteria included previous shoulder surgery, SLAP lesions with concomitant 


\section{Original article}

labral cysts, ${ }^{27}$ clinical and radiological signs of arthritis of the acromioclavicular (AC) or glenohumeral joint, ${ }^{28}$ and tears of the rotator cuff or the long head of the biceps tendon. Detailed criteria are described in the published study protocol. ${ }^{26}$

\section{Diagnostic arthroscopy}

Arthroscopic examination of the shoulder was performed in all patients under general anaesthesia, with the use of standard posterior and anterior portals. The orthopaedic surgeon (CPS) evaluated both the subacromial space and the glenohumeral joint and noted the intra-articular findings (figure 1), and a video was created for each patient.

\section{Randomisation}

Participants who fulfilled the inclusion criteria and consented to take part in the trial were randomised to receive either (1) labral repair, (2) biceps tenodesis or (3) sham surgery. An independent statistician used the permuted block method of randomisation. Concealed allocation was organised by an independent secretary who distributed sealed opaque-numbered envelopes to the head nurse in the operating room. The nurse opened the envelope when a patient was confirmed to be eligible by arthroscopic diagnostic evaluation. Following confirmation of an isolated type II SLAP lesion, the patient was included in the randomisation procedure.

\section{Interventions}

A detailed description of each intervention is provided in the published protocol. ${ }^{26}$ A single experienced shoulder surgeon performed all arthroscopic evaluations and treatments.

\section{Operative and postoperative procedures}

During the labral repair, the superior glenoid rim was debrided with a motorised shaver, followed by a percutaneous placement of a drill guide and anchors through the myotendinous

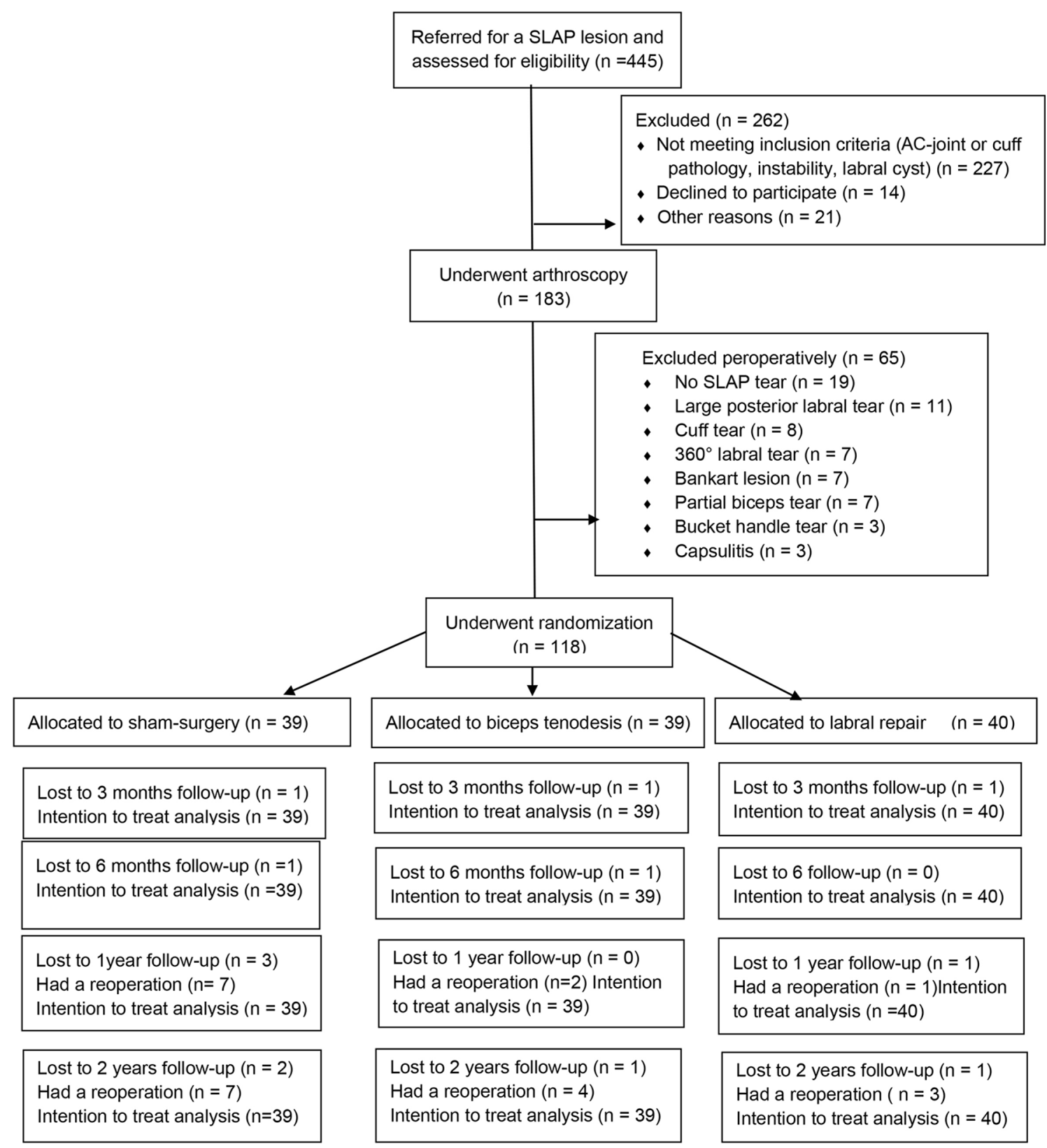

Figure 1 CONSORT (Consolidated Standards of Reporting Trials) flow chart. AC, acromioclavicular; SLAP, superior labrum anterior posterior. 
Table 1 Baseline characteristics of the patients according to study group

\begin{tabular}{|c|c|c|c|}
\hline Characteristic & $\begin{array}{l}\text { Biceps } \\
\text { tenodesis } \\
(n=39)\end{array}$ & $\begin{array}{l}\text { Sham surgery } \\
(n=39)\end{array}$ & $\begin{array}{l}\text { Labral repair } \\
(n=40)\end{array}$ \\
\hline Age (years), median (range) & 40 (18 to 64$)$ & $40(23$ to 60$)$ & 42 (22 to 57 ) \\
\hline Female sex, $\mathrm{n}(\%)$ & $15(38.5)$ & $17(42.5)$ & $15(37.5)$ \\
\hline Body mass indext & $27.6(5.6)$ & $26.1(3.8)$ & $26.4(4.3)$ \\
\hline University education, $\mathrm{n}(\%)$ & $18(48.6)$ & $23(59.0)$ & $22(56.4)$ \\
\hline Manual labour, $\mathrm{n}(\%) \ddagger$ & $19(48.7)$ & $11(28.2)$ & $17(42.5)$ \\
\hline Physical activity, n (\%)§ & $18(47.2)$ & $21(56.4)$ & $28(70.0)$ \\
\hline No training & $22(55.0)$ & $17(42.5)$ & $12(30.0)$ \\
\hline Frequent physical activity & $17(42.5)$ & $17(42.5)$ & $27(67.5)$ \\
\hline Competitive & $1(2.5)$ & $5(12.5)$ & $1(2.5)$ \\
\hline $\begin{array}{l}\text { Taking analgesics daily or } \\
\text { weekly, } \mathrm{n}(\%)\end{array}$ & $7(17.9)$ & $5(12.8)$ & $6(15.0)$ \\
\hline $\begin{array}{l}\text { Duration of pain, month } \\
\text { median (IQR) }\end{array}$ & $24(59)$ & $26(61)$ & $24(47)$ \\
\hline $\begin{array}{l}\text { Dominant shoulder involved, } \\
\mathrm{n}(\%)\end{array}$ & $28(71.8)$ & $33(84.6)$ & $28(70.0)$ \\
\hline Positive O'Brien test, $\mathrm{n}(\%) \uparrow$ & 37 (94.9) & $33(86.8)$ & $36(90.0)$ \\
\hline
\end{tabular}

*Plus-minus values are means \pm SD.

†The body mass index is the weight in kilograms divided by the square of the height in metres.

$\ddagger$ Manual labour was reported if the patient was engaged in daily manual work. §hysical activity was reported if the patient had no regular training, frequent if the patient was training two times or more in a week on a regular basis, and competitive if the patient was competing on a national level.

IThe $0^{\prime}$ Brien test is positive if the patient has pain when resisting force from an examiner, with the arm in $90^{\circ}$ of flexion, $20^{\circ}$ adduction and supinated, and less pain with the arm otherwise in the same position but with pronation.

junction of the supraspinatus. All anchors were placed posterior to the biceps root, and single circular sutures were used.

The biceps tenodesis was done with a mini-open technique. Under arthroscopic vision, a spinal needle was placed as laterally as possible and with a $90^{\circ}$ angle central in the biceps tendon and a tenotomy was performed at the biceps insertion. With less than $2 \mathrm{~cm}$ skin incision (to mimic the scars in the other two groups), the spinal needle was followed down to the biceps pulley, the pulley was split and the biceps tendon was lifted out. The groove was debrided, a double-loaded metal anchor was placed in the inferior part of the groove, and the tendon secured with two sutures, each passing twice through the tendon. The proximal part of the tendon was excised and the wound closed.

For the sham surgery, standard diagnostic shoulder arthroscopy was performed. In addition, a $5 \mathrm{~mm}$ skin incision was made to mimic a labral repair. The patient was kept in the operation theatre for the amount of time required to perform an actual arthroscopic index shoulder surgery.

All operative procedures were recorded on video. For all patients, a sling was placed before they left the operating room, and standard postoperative care and instructions were provided.

\section{Postoperative rehabilitation}

Patients in all three groups had a standardised, but individually adjusted rehabilitation. Elbow, wrist, and finger mobilisation and gentle pendulum exercises were conducted, starting on the first day. A sling was used for 3 weeks. Local physiotherapists, blinded to the allocation of the patient, provided treatment after discharge from the hospital. Passive techniques, such as massage and stretching, core stability exercises and general physical training, were used during the first
3 weeks. Exercises to improve the scapula-humeral rhythm, coordination and mobility were performed using sling exercise therapy. ${ }^{29}$ Gradual biceps loading was started at 12 weeks. Exercises to improve functional stability and scapula muscles were progressively emphasised after 6 weeks. Sports and job-specific rehabilitation were provided on an individual basis, usually starting 3 months postoperatively. Rehabilitation was continued for 3-6 months and included 12-16 sessions with a therapist and 20 self-administered exercises.

\section{Outcome measures}

The two primary outcome measures were the Rowe score ${ }^{30}$ and the Western Ontario Shoulder Instability Index (WOSI) ${ }^{31}$ at 6 and 24 months after surgery. The 1988 version of the Rowe score and the WOSI were validated for use in the present study. ${ }^{32-34}$ As recommended by Rowe, patient satisfaction was assessed separately using a self-reported question with response alternatives of poor, fair, good and excellent. ${ }^{35}$

Secondary outcomes were: WOSI and Rowe at 3 and 12 months, the Oxford Instability Shoulder Score (OISS), ${ }^{36}$ the EuroQol (EQ-5D, EQ-VAS) for generic health-related quality of $\mathrm{life}^{37}$ and change of the main symptom (pain) from baseline to all follow-ups. ${ }^{38}$ The EuroQol and OISS are validated for use in patients with SLAP lesions. ${ }^{33} 34$

A single-blinded observer assessed all participants and administered questionnaires at baseline and all postoperative follow-ups. Patient characteristics were assessed at baseline and included self-reported manual labour (yes/no); physical activity $(1=$ competitive sports $/ 2=$ frequent physical activity (three to four times a week) $/ 3=$ occasional or none); dominant shoulder (yes/no); Apprehension test (positive/negative); Crank test (positive/negative); and O'Brien test (positive/negative). Blinding was evaluated by a question asking the patients if they thought they were operated or had sham surgery. Adverse events were classified as serious if the patient needed prolonged inpatient hospital care, hospitalisation or death, and considerable if the patient had painful shoulder stiffness (capsulitis) with external rotation and forward flexion $<30^{\circ}$ and abduction $<90^{\circ}$. $^{39}$

\section{Sample size and statistical analysis}

The study was designed to detect a clinically important detectable difference of 10 points in mean Rowe score. To detect this difference among treatment groups $(S D=15, \alpha=0.05$, $\beta=0.80$, one-way analysis of variance), the study required 36 patients in each group. Assuming 10\% dropout, we planned to include 40 patients in each group. In a later study, ${ }^{32}$ we found the minimal clinically important change for Rowe score to be 17. At 6 months there were no crossovers and the results were analysed and interpreted blindly, as recommended by Järvinen et al. ${ }^{40}$

Continuous variables are presented as mean values with 95\% CI and categorical values as numbers and percentages. We used a mixed models approach to analyse the effect of the interventions on primary and secondary variables at 6 and 24 months. For each model, we included a core set of adjustment variables as covariates: age, sex, time, frequency of physical activity, manual labour and dominant arm. In addition, the baseline measurement was included as a covariate to eliminate regression towards the mean for the estimated changes over time of the different treatments and for pairwise comparisons. The final model was tested for confounding with the covariates not included in the final main effects model. Confounding 


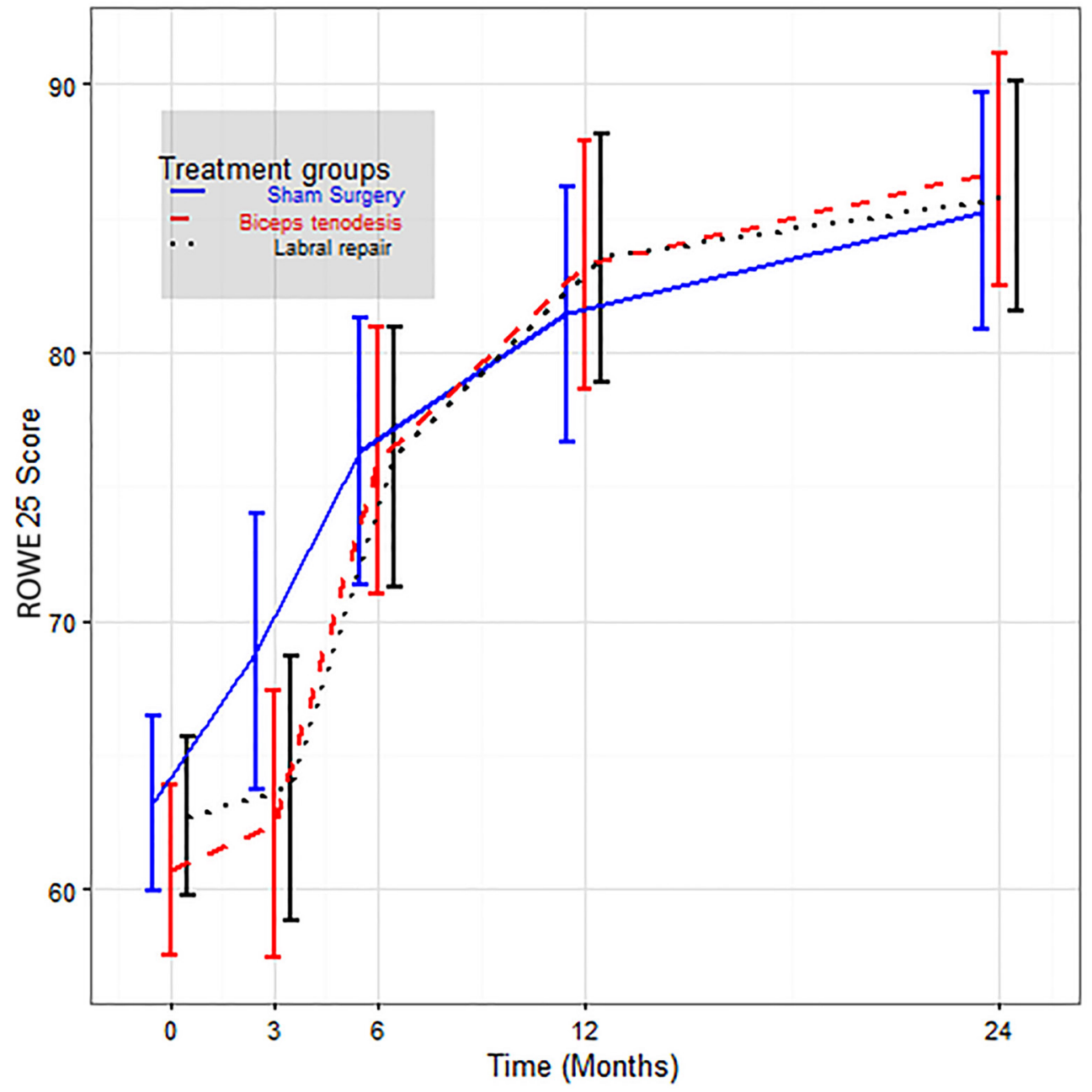

Figure 2 Rowe scores (with error bars) for each group at baseline and at 3, 6, 12 and 24 months.

was defined as a change in the estimate of at least $25 \%$ when a term was added to a model. Finally, we tested for interactions between covariates and all outcomes at the 0.05 level only. If a significant difference was found, we applied Tukey's test for multiple comparisons. The assumptions were explored using Cook's distance, covariance ratio and trace statistics to assess the validity of the model. To ascertain the robustness of findings, we performed a bootstrap regression analysis with 1000 replications, adjusting for the baseline values and the variables mentioned above. Likewise, we performed multiple imputation using the Markov chain Monte Carlo method. The analysis was performed with the missing data as is and with the imputed values. For categorical variables, we applied the mixed models with a multinomial distribution. For sensitivity analysis, we performed per protocol analysis comparing results in those who adhered to the protocol. Post hoc analyses were used to compare primary outcome scores at 6 months in those who crossed over from sham surgery with scores in a similar number of patients with the most inferior scores of patients randomised to biceps tenodesis and labral repair. The analyses were performed in Statistical Analysis System (SAS, V.9.4) and R V.3.1.1.

\section{Ethics and registration}

Ethics approval (IRB00001870) was received from the Ethics Committee Health Region Southeast, Oslo, Norway. The study was conducted in accordance with the Declaration of
Helsinki. All patients provided written informed consent. They were informed that they might undergo sham surgery and that their group assignment could be unblinded after 6 months if they were not satisfied with their shoulder function. The study was also registered with ClinicalTrials.gov (NCT00586742).

\section{RESULTS}

\section{Patient characteristics}

Of the 445 patients eligible for inclusion, 262 were excluded before arthroscopy. Of the 183 patients who had arthroscopy, 65 were excluded peroperatively (figure 1). A total of 118 patients were randomised; 39 were assigned to sham surgery, 39 to biceps tenodesis and 40 to labral repair. The mean age was 40 years, ranging from 18 to 64. One patient aged 64 years was mistakenly included. The baseline characteristics are described in table 1.

Four patients were lost to follow-up at 2 years; two in the sham group and one in each of the other two groups.

\section{Primary outcomes}

There was a significant improvement from baseline to 6 and 24 months for all three study groups (figure 2 and table 2). Age, sex, trauma, manual work, frequency of physical activity, duration of pain and dominant shoulder were not significantly associated with primary outcomes at follow-up. The only 
Table 2 Primary and secondary outcomes at baseline and at 3, 6, 12 and 24 months after arthroscopy*

\begin{tabular}{|c|c|c|c|}
\hline Outcome & $\begin{array}{l}\text { Biceps tenodesis } \\
(n=39)\end{array}$ & $\begin{array}{l}\text { Sham surgery } \\
(n=39)\end{array}$ & $\begin{array}{l}\text { Labral repair } \\
(n=40)\end{array}$ \\
\hline \multicolumn{4}{|c|}{ Primary outcome, mean (95\% Cl) } \\
\hline \multicolumn{4}{|c|}{ Rowe score (0 to 100) $\dagger$} \\
\hline Baseline & $60.3(57.0$ to 64.0$)$ & 63.2 (59.8 to 66.7$)$ & 62.7 (59.6 to 65.8$)$ \\
\hline 3 Months & 62.4 (57.1 to 67.7$)$ & $68.9(63.5$ to 74.3$)$ & 63.8 (58.6 to 69.0$)$ \\
\hline 6 Months & 76.0 (70.7 to 81.1$)$ & 76.3 (71.1 to 81.6$)$ & 76.1 (71.0 to 81.3$)$ \\
\hline 12 Months & 83.3 (78.4 to 88.1$)$ & 81.4 (76.4 to 86.5$)$ & 83.5 (78.6 to 88.4$)$ \\
\hline 24 Months & 86.8 (82.2 to 91.4$)$ & 85.3 (80.6 to 89.9$)$ & 85.8 (81.3 to 90.4$)$ \\
\hline \multicolumn{4}{|c|}{ WOSI (0 to 2100$) \ddagger$} \\
\hline Baseline & 1155 (1026 to 1283) & 1062 (940 to 1183 ) & 1044 (941 to 1146$)$ \\
\hline 3 Months & 1115 (829 to 1401) & 833 (528 to 1138 ) & 788 (506 to 1070$)$ \\
\hline 6 Months & 689 (548 to 829$)$ & 560 (420 to 701$)$ & 552 (414 to 691$)$ \\
\hline 12 Months & 490 (353 to 627$)$ & 475 (335 to 614$)$ & 429 (294 to 565$)$ \\
\hline 24 Months & 436 (313 to 559$)$ & 458 (334 to 582$)$ & $340(219$ to 461$)$ \\
\hline \multicolumn{4}{|c|}{ Secondary outcome, mean $(95 \% \mathrm{Cl})$} \\
\hline \multicolumn{4}{|c|}{ OISS (12 to 60$) \S$} \\
\hline Baseline & 38.6 (36.1 to 41.1$)$ & 36.9 (34.3 to 39.6$)$ & 36.7 (34.8 to 38.6$)$ \\
\hline 3 Months & $37.8(35.3$ to 40.4$)$ & 36.0 (33.4 to 38.6$)$ & 36.0 (33.6 to 38.5 ) \\
\hline 6 Months & 29.7 (27.0 to 32.4$)$ & 28.6 (25.9 to 31.3$)$ & 27.4 (24.7 to 30.60 \\
\hline 12 Months & 24.1 (21.1 to 27.1 ) & 25.9 (22.8 to 28.9$)$ & 23.3 (20.3 to 26.2 ) \\
\hline 24 Months & 21.7 (18.5 to 24.8$)$ & 23.3 (20.1 to 26.5$)$ & 22.7 (19.6 to 25.8$)$ \\
\hline \multicolumn{4}{|c|}{ EQ-5D (0.59 to 1.00$)$ q } \\
\hline Baseline & $0.6(0.5$ to 0.7$)$ & $0.7(0.6$ to 0.7$)$ & 0.7 (0.6 to 0.7$)$ \\
\hline 3 Months & $0.6(0.6$ to 0.7$)$ & 0.7 (0.7 to 0.8$)$ & 0.7 (0.6 to 0.8$)$ \\
\hline 6 Months & 0.7 (0.7 to 0.8$)$ & $0.8(0.8$ to 0.9$)$ & $0.8(0.7$ to 0.9$)$ \\
\hline 12 Months & $0.8(0.8$ to 0.9$)$ & $0.8(0.7$ to 0.9$)$ & $0.8(0.8$ to 0.9$)$ \\
\hline 24 Months & $0.8(0.8$ to 0.9$)$ & $0.9(0.8$ to 0.9$)$ & $0.9(0.8$ to 0.9$)$ \\
\hline \multicolumn{4}{|c|}{ EQ-VAS $(0$ to 100$)$ ๆ } \\
\hline Baseline & $67.9(63.3$ to 73.4$)$ & 66.7 (62.2 to 73.1$)$ & 68.4 (62.9 to 73.8$)$ \\
\hline 3 Months & 67.1 (60.7 to 73.5$)$ & 67.7 (61.6 to 74.3$)$ & 72.9 (66.6 to 79.3$)$ \\
\hline 6 Months & 73.9 (68.3 to 79.4$)$ & 76.9 (71.3 to 82.4$)$ & 81.3 (75.8 to 86.7$)$ \\
\hline 12 Months & 78.3 (72.5 to 84.1$)$ & 79.4 (73.5 to 85.3$)$ & 79.2 (73.5 to 85.0$)$ \\
\hline 24 Months & 79.6 (74.0 to 85.2$)$ & 76.8 (71.2 to 82.5$)$ & 81.7 (76.1 to 87.2$)$ \\
\hline \multicolumn{4}{|c|}{ Change of main complaint $(-9$ to +9$)$} \\
\hline 3 Months & $3.0(1.5$ to 4.5$)$ & $2.3(0.8$ to 3.9$)$ & $3.2(1.7$ to 4.6$)$ \\
\hline 6 Months & $3.8(2.2$ to 5.4$)$ & $2.8(1.2$ to 4.4$)$ & $3.7(2.1$ to 5.3$)$ \\
\hline 12 Months & 5.3 (3.9 to 6.6$)$ & 4.5 (3.1 to 5.9$)$ & $5.5(4.2$ to 7.0$)$ \\
\hline 24 Months & $5.7(4.6$ to 6.9$)$ & 5.5 (4.3 to 6.7$)$ & $5.3(4.3$ to 6.9$)$ \\
\hline
\end{tabular}

*Unadjusted values are given.

tHigher scores indicating better shoulder function.

$\ddagger$ Western Ontario Shoulder Instability Index, lower scores indicating better shoulder function.

$\S 0 x f o r d$ Instability Shoulder Score, lower scores indicating better shoulder function.

१EuroQol (EQ-5D and EQ-VAS), higher scores indicating better health-related quality of life.

OISS, Oxford Instability Shoulder Score; WOSI, Western Ontario Shoulder Instability Index.

significant covariates were the baseline Rowe $(\mathrm{p}<0.001)$ and WOSI $(\mathrm{p}<0.002)$ scores.

There were no significant between-group differences in the change from baseline to 6 or 24 months in any primary outcome, neither for the adjusted nor for the unadjusted results (table 3). Unadjusted results were marginally different and imputation did not materially change between-group differences.

\section{Secondary and other outcomes}

All three groups improved on the secondary outcomes from baseline to 6 and 24 months (table 2), and there were no significant differences among the groups (table 3 ). In the sham group, 31/37 (84\%) patients reported excellent or good results at 1 -year follow-up, in the labral repair and biceps tenodesis groups the numbers were 29/35 (83\%) and 34/38 (89\%), respectively. The first 25 patients did not receive the blinding question. After sham surgery, 19/26 (73\%) patients believed they were repaired, in comparison to $31 / 32$ (97\%) after biceps tenodesis and 31/35 (89\%) after labral repair.

There were no serious adverse events, infections or nerve injuries. Ten patients experienced prolonged postoperative stiffness (capsulitis); five after labral repair, four after biceps tenodesis and one after sham surgery (due to trauma 3 weeks postoperatively).

\section{Per protocol analysis}

There were no significant differences in the changes between groups for any primary or secondary outcomes in the per protocol analysis. At 6 and 24 months, respectively, the mean 
Table 3 Adjusted between-group differences at 3, 6, 12 and 24 months after arthroscopy*

\begin{tabular}{|c|c|c|c|c|c|c|}
\hline Outcome & $\begin{array}{l}\text { Biceps tenodesis versus } \\
\text { labral repair }\end{array}$ & $\mathrm{p}$ Value & $\begin{array}{l}\text { Biceps tenodesis versus } \\
\text { sham surgery }\end{array}$ & $p$ Value & $\begin{array}{l}\text { Labral repair versus } \\
\text { sham surgery }\end{array}$ & $\mathrm{p}$ Value \\
\hline \multicolumn{7}{|c|}{ Primary outcomes } \\
\hline \multicolumn{7}{|c|}{ Rowe score (0 to 100)† } \\
\hline 3 Months & $-1.3(-8.7$ to 6.0$)$ & .76 & $-6.5(-14.0$ to 1.1$)$ & .09 & $-5.1(-12.6$ to 2.4$)$ & .18 \\
\hline 6 Months & $-0.2(-7.5$ to 7.1$)$ & .96 & $-0.2(-7.8$ to 7.0$)$ & .92 & $-0.2(-7.5$ to 7.1$)$ & .95 \\
\hline 12 Months & $-0.2(-7.1$ to 6.6$)$ & .95 & $1.8(-5.2$ to 8.8$)$ & .61 & 2.1 (-5.9 to 9.0$)$ & .56 \\
\hline 24 Months & $1.0(-5.4$ to 7.4$)$ & .76 & $1.6(-5.0$ to 8.1$)$ & .64 & 0.6 (-5.9 to 7.0$)$ & .86 \\
\hline \multicolumn{7}{|c|}{ WOSI (0 to 2100)‡ } \\
\hline 3 Months & $-327(-728$ to 75$)$ & .11 & $-282(-700$ to 137$)$ & .19 & $45(-370$ to 461$)$ & .83 \\
\hline 6 Months & $-137(-335$ to 61$)$ & .17 & $-128(-328$ to 71$)$ & .20 & 8 (-189 to 205$)$ & .93 \\
\hline 12 Months & $-60(-254$ to 133$)$ & .53 & $-15(-211$ to 181$)$ & .88 & 45 (-149 to 240$)$ & .64 \\
\hline 24 Months & $-96(-269$ to 77$)$ & .27 & $22(-152$ to 196$)$ & .80 & $118(-54$ to 291$)$ & .18 \\
\hline \multicolumn{7}{|c|}{ Secondary outcomes } \\
\hline \multicolumn{7}{|c|}{ OISS (12 to 60$) \S$} \\
\hline 3 Months & $-1.8(-5.4$ to 1.8$)$ & .32 & $-1.8(-5.4$ to 1.8$)$ & .32 & 0.0 (-3.6 to 3.6$)$ & .99 \\
\hline 6 Months & -2.3 (6.1 to 1.5$)$ & .56 & -1.1 ( -5.0 to 2.7$)$ & .22 & 1.2 (-5.0 to 2.6$)$ & .53 \\
\hline 12 Months & $-0.8(-5.4$ to 5.0$)$ & .41 & $1.8(-2.3$ to 6.5$)$ & .41 & 2.6 (-1.6 to 6.9$)$ & .23 \\
\hline 24 Months & $1.0(-5.5$ to 3.5$)$ & .65 & $1.6(-2.9$ to 2.3$)$ & .65 & $0.6(-3.9$ to 5.1$)$ & .79 \\
\hline \multicolumn{7}{|c|}{ EQ-5D $(-0.59$ to 1.00$)$ व } \\
\hline 3 Months & $-0.1(-0.2$ to 0.0$)$ & .18 & $-0.1(-0.2$ to 0.0$)$ & .03 & $-0.1(-0.1$ to 0.1$)$ & .41 \\
\hline 6 Months & $-0.1(-0.1$ to 0.0$)$ & .19 & $-0.1(-0.2$ to 0.0$)$ & .08 & $0.0(-0.1$ to 0.1$)$ & .65 \\
\hline 12 Months & $0.0(-0.1$ to 0.1$)$ & .85 & $0.0(0.0$ to 0.1$)$ & .37 & $0.0(-0.1$ to 0.1$)$ & .47 \\
\hline 24 Months & $0.0(-0.1$ to 0.1$)$ & .50 & $0.0(-0.1$ to 0.1$)$ & .56 & $0.0(-0.1$ to 0.0$)$ & .93 \\
\hline \multicolumn{7}{|c|}{ EQ-VAS $(0$ to 100$)$ ๆ } \\
\hline 3 Months & $-5.8(-14.8$ to 3.2$)$ & .20 & $-0.6(-9.8$ to 8.5$)$ & .90 & $5.2(-4.0$ to 14.4$)$ & .26 \\
\hline 6 Months & $-7.4(-15.2$ to 0.4$)$ & .06 & $-3.1(-10.9$ to 4.8$)$ & .44 & $4.4(-3.4$ to -12.1$)$ & .27 \\
\hline 12 Months & $-1.0(-9.2$ to 7.2$)$ & .82 & $-1.1(-9.4$ to 7.1$)$ & .79 & $-0.2(-8.4$ to 8.1$)$ & .97 \\
\hline 24 Months & $-2.1(-10.0$ to 5.8$)$ & .50 & $2.8(-5.5$ to 10.7$)$ & .49 & $4.9(-3.0$ to -12.8$)$ & .23 \\
\hline \multicolumn{7}{|c|}{ Main complaint $(-9$ to +9$)$} \\
\hline 3 Months & $-0.2(-2.3$ to 1.9$)$ & .87 & 0.6 (-1.5 to 2.8$)$ & .55 & 0.8 (-1.3 to 2.9$)$ & .44 \\
\hline 6 Months & 0.1 (-2.2 to 2.4$)$ & .93 & $1.0(-1.3$ to 3.3$)$ & .40 & $0.9(-1.4$ to 3.1$)$ & .44 \\
\hline 12 Months & $-0.1(-2.0$ to 1.8$)$ & .90 & 0.8 (-1.1 to 2.8$)$ & .41 & $1.0(-0.9$ to 2.9$)$ & .34 \\
\hline 24 Months & $-0.1(-2.0$ to 1.8$)$ & .95 & $0.2(-1.4$ to 1.9$)$ & .80 & $0.3(-1.4$ to 1.3$)$ & .75 \\
\hline
\end{tabular}

The values are expressed as mean $(95 \% \mathrm{Cl})$.

*Adjusted for age, sex, baseline score, time, manual labour, physical activity and dominant shoulder. Between-group differences were not significant for any outcome. Time and baseline score significantly predicted outcome.

tHigher Rowe scores indicating better shoulder function.

¥Western Ontario Shoulder Instability Index, lower scores indicating better shoulder function.

$\S 0 x f o r d$ Instability Shoulder Score, lower score indicating better shoulder function.

IEuroQol (EQ-5D and EQ-VAS), higher scores indicating better health-related quality of life.

OISS, Oxford Instability Shoulder Score; WOSI, Western Ontario Shoulder Instability Index.

Rowe scores for the three groups were: biceps tenodesis 76.1 and 87.0; labral repair 76.2 and 85.9; sham surgery 81.4 and 89.0. The mean WOSI scores were: biceps tenodesis 690 and 437; labral repair 552 and 339; sham surgery 428 and 338.

\section{Crossovers}

Fourteen patients in the sham group were reoperated between the 6 and 24 months of follow-up, 12 with labral repair and 2 with a biceps tenodesis. Six patients in the biceps tenodesis and four in the labral repair group were reoperated; two patients had capsular release, three had a labral repair and one patient had an AC joint resection in the tenodesis group, while three biceps tenodesis and one AC joint resection were performed in the labral repair group. The mean Rowe and WOSI scores at 6 and 24 months in the 14 patients who crossed over from the sham group were not significantly different from the 14 patients with the lowest scores in the labral repair and biceps tenodesis groups. At 24 months of follow-up, 8 of the 14 patients in the crossover group had a Rowe score over 80 and 7 of them rated the shoulder as good or excellent.

\section{DISCUSSION}

In this three-armed randomised, double-blind, sham-controlled trial comparing labral repair, biceps tenodesis and sham surgery for symptomatic, isolated SLAP II lesions of the shoulder, we found a significant improvement in objective and subjective shoulder scores for all three groups, but no significant group differences at either the 6 or 24 months of follow-up. As there is no previous sham-controlled trial of the clinical effectiveness of shoulder surgery, we discuss the results of the present sham surgical trial in the context of sham surgical trials in knee patients with knee pain. ${ }^{41} 42$

\section{Clinically relevant outcome measures}

The choice of outcome measures is an important issue. The scores used in this study were validated for use on patients 
with a SLAP lesion ${ }^{32-34}$ and capture the most important aspects of shoulder function. ${ }^{43}$ Both the clinician-based and the patient-based scores showed comparable results and differences between groups were much smaller than the different scores are able to detect.

\section{Potential limitations}

Issues related to sample size, blinding, the population studied and external validity are possible limitations of the present study. This is a small study, but the CIs of the observed between-group differences indicate that the study was adequately powered to detect clinically relevant differences between the three groups. For Rowe scores, the CIs did not include 10, which the trial was designed to detect. That only $73 \%$ of patients in the sham group believed that they had been repaired may have biased the results. Unfortunately, data did not provide enough information to calculate a blinding index. ${ }^{44}$ The primary follow-up time of 6 months was selected mainly for ethical reasons. Given that the patients were referred for surgery and that we hypothesised better results after surgical repair compared with sham surgery, it was our opinion that it would be unethical to maintain patient blinding much beyond 6 months. Therefore, we advised patients that it would take time to recover from surgery and informed them that it was our aim to maintain the blinding as long as possible. However, if they were not satisfied by 6 months, their group assignment could be unblinded.

Our results at 2 years confirm the 6-month results with no statistically significant differences between the three groups. In addition, the comparison of crossovers with a similar number of patients with inferior results in the biceps tenodesis and labral repair groups suggests that the threshold for reoperation was lower in the sham group. However, it cannot be ruled out that the higher rate of reoperation in the sham group was related to unblinding rather than to differences in treatment failure. Even though there was no significant difference in mean Rowe score in the crossover group after reoperation, it is worth noting that 8 of the 14 patients had a Rowe score over 80 .

We assessed 445 patients and included 118 according to strict inclusion criteria. This introduces a risk of selection bias but improves the internal validity of the study in order to assess isolated SLAP II lesions. Both athletes and patients with work-related injuries were included. Results were adjusted for frequency of physical activity and manual labour, but do not apply to a specific population. Future studies should compare non-operative and operative treatments in athletes, including return to sports in addition to the validated outcomes applied in the present study.

The choice of method regarding the biceps tenodesis may be questioned. Surgeons currently use several methods, both arthroscopic and open, with a variety of fixation devices. ${ }^{45} 46$ The point of fixation of the biceps tendon may be in the bicipital groove, subpectoral humeral fixation or transferred to the conjoint tendon. We fixed the biceps with a mini-open technique using a suture anchor low in the bicipital groove. Systematic reviews and randomised studies comparing different tenodesis techniques have not shown any differences in outcome. ${ }^{45} 46$

\section{Strengths of this study}

The study has several strengths: randomisation; inclusion of a sham surgery control; blinded assessments, analysis and interpretation of results; validated outcome measures; no crossovers at 6 months; and minimal loss to follow-up. Strict inclusion criteria were applied to increase the internal validity for evaluating the efficacy of the surgical procedures. The use of locally available physiotherapists and manual therapists for the postoperative exercise programme of these patients enhances the external validity of the study.

The physiotherapy provided to all groups (the sole treatment in the sham group) followed current guidelines. ${ }^{47}$ However, the study does not provide information about the effectiveness of physiotherapy with supervised exercises without diagnostic arthroscopy. Ideally, the study should have included an arm with patients not receiving any treatment (natural course), or an arm with physiotherapy without placebo surgery. We discussed this and decided that the inclusion of a fourth group would be too difficult to implement. Although physiotherapy might have contributed to the results in all groups, the impact of placebo, the natural course and regression to the mean should not be underestimated.

\section{Clinical implications}

The results of our trial extend previous reports ${ }^{12} 19$ of possible overtreatment of SLAP lesions and indicate a need to narrow indications. Patient age is debated and authors advocate that SLAP repair should be reserved for the young and active patient. ${ }^{1148}$ There were no significant differences in function, patient satisfaction or complications by age in this study, but the groups are too small to perform subgroup analysis and identify factors associated with failures. The present study does not support either labral repair or biceps tenodesis for type II SLAP lesions in this population, as we found no significant difference between treatments in any outcome. Considering the lack of high-quality trials in this field, the results of this study should be interpreted with caution.

Based on this study, we believe future patients should be informed about the long recovery and possible complications after surgery, and that non-operative treatment has a good probability of success. Future studies should evaluate non-operative treatments and predictors of success.

We conclude that at 6 and 24 months of follow-up, there were no significant differences between labral repair, biceps tenodesis and sham surgery for patients with a type II SLAP lesion in the population studied.

\section{What are the findings?}

Labral repair, biceps tenodesis and sham surgery for patients with an isolated SLAP II lesion all led to significant improvement in both objective and subjective scores. There was no significant difference among (1) labral repair, (2) biceps tenodesis and (3) sham surgery in the population studied.

\section{How might it impact on clinical practice in the future?}

Patients should be told that non-operative treatment has a good probability of success, but further studies are needed to establish what treatment is the best for the young active patient.

Acknowledgements We thank Lovisenberg Diaconal Hospital, Lars Vasli, Chief of the Surgical Department, all the patients, the referring physicians, the nurses assisting in the operating theatre, the physiotherapists and manual therapists conducting the postoperative physiotherapy, Jostein Skranes Brox (blinded to the treatment given) for entering the data, and Caryl Gay for linguistic comments on the final manuscript.

Contributors CPS, OR and JIB conceived and designed the study. ØS and PM participated in setting up of the study. ØS participated in patient recruitment and data collection. PM conducted the analysis and, together with JIB, ØS, OR and CPS, interpretation. CPS and JIB drafted the first version of the manuscript. All authors helped revise the manuscript and gave their final approval of the submitted version. 
Competing interests None declared.

Ethics approval This study was approved (IRB00001870) by the Ethics Commitee Health Region Southeast, Oslo, Norway.

Provenance and peer review Not commissioned; externally peer reviewed.

Data sharing statement Data are available on request.

Open Access This is an Open Access article distributed in accordance with the Creative Commons Attribution Non Commercial (CC BY-NC 4.0) license, which permits others to distribute, remix, adapt, build upon this work non-commercially, and license their derivative works on different terms, provided the original work is properly cited and the use is non-commercial. See: http://creativecommons.org/ licenses/by-nc/4.0/

(c) Article author(s) (or their employer(s) unless otherwise stated in the text of the article) 2017. All rights reserved. No commercial use is permitted unless otherwise expressly granted.

\section{REFERENCES}

1 Snyder SJ, Karzel RP, Del Pizzo W, et al. SLAP lesions of the shoulder. Arthroscopy 1990;6:274-9.

2 Olsson 0. Degenerative changes of the shoulder joint and their connection with shoulder pain. PhD Thesis, University of Gothenborg. 1953.

3 Hegedus EJ, Goode AP, Cook CE, et al. Which physical examination tests provide clinicians with the most value when examining the shoulder? update of a systematic review with meta-analysis of individual tests. Br J Sports Med 2012;46:964-78.

4 Amin MF, Youssef AO. The diagnostic value of magnetic resonance arthrography of the shoulder in detection and grading of SLAP lesions: comparison with arthroscopic findings. Eur J Radiol 2012;81:2343-7.

5 Gobezie R, Zurakowski D, Lavery K, et al. Analysis of interobserver and intraobserver variability in the diagnosis and treatment of SLAP tears using the snyder classification. Am J Sports Med 2008:36:1373-9.

6 Holzapfel K, Waldt S, Bruegel M, et al. Inter- and intraobserver variability of MR arthrography in the detection and classification of superior labral anterior posterior (SLAP) lesions: evaluation in 78 cases with arthroscopic correlation. Eur Radiol 2010;20:666-73.

7 Jia X, Yokota A, McCarty EC, et al. Reproducibility and reliability of the Snyder classification of superior labral anterior posterior lesions among shoulder surgeons. Am J Sports Med 2011:39:986-91.

8 Sheridan K, Kreulen C, Kim S, et al. Accuracy of magnetic resonance imaging to diagnose superior labrum anterior-posterior tears. Knee Surg Sports Traumatol Arthrosc 2015;23:2645-50

9 Schwartzberg R, Reuss BL, Burkhart BG, et al. High prevalence of superior labral tears diagnosed by MRI in middle-aged patients with asymptomatic shoulders. Orthop J Sports Med 2016;5:1-7.

10 Katz LM, Hsu S, Miller SL, et al. Poor outcomes after SLAP repair: descriptive analysis and prognosis. Arthroscopy 2009;25:849-55.

11 Vogel LA, Moen TC, Macaulay AA, et al. Superior labrum anterior-to-posterior repair incidence: a longitudinal investigation of community and academic databases. J Shoulder Elbow Surg 2014;23:e119-e126.

12 Weber SC, Martin DF, Seiler JG, et al. Superior labrum anterior and posterior lesions of the shoulder: incidence rates, complications, and outcomes as reported by American Board of Orthopedic Surgery. Part II candidates. Am I Sports Med 2012;40:1538-43.

13 Boileau P, Parratte S, Chuinard C, et al. Arthroscopic treatment of isolated type II SLAP lesions: biceps tenodesis as an alternative to reinsertion. Am I Sports Med 2009;37:929-36.

14 Brockmeier SF, Voos JE, Williams RJ, et al; Hospital for Special Surgery Sports Medicine and Shoulder Service. Outcomes after arthroscopic repair of type-II SLAP lesions. J Bone Joint Surg Am 2009;91:1595-603.

15 Gorantla K, Gill C, Wright RW. The outcome of type II SLAP repair: a systematic review. Arthroscopy 2010;26:537-45.

16 Provencher MT, McCormick F, Dewing C, et al. A prospective analysis of 179 type 2 superior labrum anterior and posterior repairs. Am I Sports Med 2013:41:880-6

17 Schrøder CP, Skare O, Gjengedal E, et al. Long-term results after SLAP repair: a 5-year follow-up study of 107 patients with comparison of patients aged over and under 40 years. Arthroscopy 2012;28:1601-7.

18 Erickson BJ, Jain A, Abrams GD, et al. SLAP lesions: trends in treatment. Arthroscopy 2016:32:976-81.

19 Patterson BM, Creighton RA, Spang JT, et al. Surgical trends in the treatment of superior labrum anterior and posterior lesions of the shoulder: analysis of data from the American board of orthopaedic surgery certification examination database. Am J Sports Med 2014:42:1904-10.

20 Gupta AK, Chalmers PN, Klosterman EL, et al. Subpectoral biceps tenodesis for bicipital tendonitis with SLAP tear. Orthopedics 2015;38:e48-e53.
21 Ek ET, Shi LL, Tompson JD, et al. Surgical treatment of isolated type II superior labrum anterior-posterior (SLAP) lesions: repair versus biceps tenodesis. J Shoulder Elbow Surg 2014;23:1059-65.

22 Gottschalk MB, Karas SG, Ghattas TN, et al. Subpectoral biceps tenodesis for the treatment of type II and IV superior labral anterior and posterior lesions. Am J Sports Med 2014:42:2128-35.

23 Edwards SL, Lee JA, Bell JE, et al. Nonoperative treatment of superior labrum anterior posterior tears: improvements in pain, function, and quality of life. Am J Sports Med 2010;38:1456-61.

24 Jang $\mathrm{SH}$, Seo JG, Jang $\mathrm{HS}$, et al. Predictive factors associated with failure of nonoperative treatment of superior labrum anterior-posterior tears. J Shoulder Elbow Surg 2016;25:428-34.

25 Fedoriw WW, Ramkumar P, McCulloch PC, et al. Return to play after treatment of superior labral tears in professional baseball players. Am I Sports Med 2014:42:1155-60.

26 Skare 0 , Schrøder CP, Reikerås 0 , et al. Efficacy of labral repair, biceps tenodesis, and diagnostic arthroscopy for SLAP lesions of the shoulder: a randomised controlled trial. BMC Musculoskelet Disord 2010;11:228.

27 Schroder CP, Skare O, Stiris M, et al. Treatment of labral tears with associated spinoglenoid cysts without cyst decompression. J Bone Joint Surg Am 2008;90:523-30.

28 Walton J, Mahajan S, Paxinos A, et al. Diagnostic values of tests for acromioclavicular joint pain. J Bone Joint Surg Am 2004;86-A:807-12.

29 Bøhmer AS, Staff PH, Brox Jl. Supervised exercises in relation to rotator cuff disease (impingement syndrome stages II and III): A treatment regimen and its rationale. Physiother Theory Pract 1998;14:93-105.

30 Rowe CR. Evaluation of the shoulder. Rowe CR, ed. The shoulder. New York: churchill livingstone, 1988

31 Kirkley A, Griffin S, McLintock H, et al. The development and evaluation of a diseasespecific quality of life measurement tool for shoulder instability. The Western Ontario Shoulder Instability Index (WOSI). Am J Sports Med 1998;26:764-72.

32 Skare $\varnothing$, Schrøder CP, Mowinckel $P$, et al. Reliability, agreement and validity of the 1988 version of the rowe score. J Shoulder Elbow Surg 2011:20:1041-9.

33 Skare $\varnothing$, Mowinckel P, Schrøder CP, et al. Responsiveness of outcome measures in patients with superior labral anterior and posterior lesions. Shoulder Elbow 2014;6:262-72.

34 Skare $\varnothing$, Liavaag S, Reikerås 0 , et al. Evaluation of Oxford instability shoulder score, western ontario shoulder instability index and euroqol in patients with SLAP (superior labral anterior posterior) lesions or recurrent anterior dislocations of the shoulder. BMC Res Notes 2013;6:273.

35 Rowe $C R$, Patel $D$, Southmayd WW. The bankart procedure: a long-term end-result study. J Bone Joint Surg Am 1978;60:1-16.

36 Dawson J, Fitzpatrick R, Carr A. The assessment of shoulder instability. The development and validation of a questionnaire. J Bone Joint Surg Br 1999;81:420-6.

37 EuroQol Group. EuroQol--a new facility for the measurement of health-related quality of life. Health Policy 1990;16:199-208.

38 Brox JI, Gjengedal E, Uppheim G, et al. Arthroscopic surgery versus supervised exercises in patients with rotator cuff disease (stage II impingement syndrome): a prospective, randomized, controlled study in 125 patients with a $21 / 2$-year follow-up. J Shoulder Elbow Surg 1999;8:102-11.

39 Tveitå EK, Tariq R, Sesseng S, et al. Hydrodilatation, corticosteroids and adhesive capsulitis: a randomized controlled trial. BMC Musculoskelet Disord 2008:9:9-53.

40 Järvinen TL, Sihvonen R, Bhandari M, et al. Blinded interpretation of study results can feasibly and effectively diminish interpretation bias. J Clin Epidemiol 2014;67:769-72.

41 Moseley JB, O'Malley K, Petersen NJ, et al. A controlled trial of arthroscopic surgery for osteoarthritis of the knee. N Engl I Med 2002;347:81-8.

42 Sihvonen R, Paavola M, Malmivaara A, et al; Finnish Degenerative Meniscal Lesion Study (FIDELITY) Group. Arthroscopic partial meniscectomy versus sham surgery for a degenerative meniscal tear. N Engl I Med 2013;369:2515-24.

43 Roe Y, Østensjø S. Conceptualization and assessment of disability in shoulder-specific measures with reference to the international classification of functioning, disability and health. J Rehabil Med 2016;48:325-32.

44 Bang H, Ni L, Davis CE. Assessment of blinding in clinical trials. Control Clin Trials 2004:25:143-56

45 Abraham VT, Tan BH, Kumar VP. Systematic review of biceps tenodesis: arthroscopic versus open. Arthroscopy 2016;32:365-71.

46 Green JM, Getelman MH, Snyder SJ, et al. All-Arthroscopic suprapectoral versus open subpectoral tenodesis of the long head of the biceps brachii without the use of interference screws. Arthroscopy 2017;33:19-25.

47 Cools AM, Borms D, Cottens S, et al. Rehabilitation exercises for athletes with biceps disorders and SLAP lesions: a continuum of exercises with increasing loads on the biceps. Am J Sports Med 2014;42:1315-22.

48 Burns JP, Bahk M, Snyder SJ. Superior labral tears: repair versus biceps tenodesis. Shoulder Elbow Surg 2011;20:S2-S8. 